\title{
Delayed Subdural Hematoma After Epidural Blood Patching in a Patient With Spontaneous Intracranial Hypotension -Case Report-
}

\author{
Hao-Kuang WANG, Po-Chou LILIANG, Cheng-Loong LiANG, \\ Kang LU, Kuo-Chuan HUNG*, and Han-Jung CHEN \\ Department of Neurosurgery and * Anesthesiology, E-Da Hospital, \\ I-Shou University, Kaohsiung, Taiwan
}

\begin{abstract}
A 51-year-old woman presented with a rare case of spontaneous intracranial hypotension (SIH) complicated by a delayed subdural hematoma (SDH) that required surgical evacuation 2 months after epidural blood patching (EBP). Subdural fluid collections are common among patients with SIH. These fluid collections vary in appearance from thin subdural hygromas to rare large SDHs associated with significant mass effect. Most subdural fluid collections can be safely managed by conservative treatment or EBP. The present clinical course and imaging findings illustrate the possible sequential complications of EBP in patients with SIH.
\end{abstract}

Key words: subdural hematoma, epidural blood patching, spontaneous intracranial hypotension, cerebrospinal fluid, magnetic resonance cisternography

\section{Introduction}

Orthostatic headache is a common presentation of spontaneous intracranial hypotension (SIH), which is associated with cerebrospinal fluid (CSF) leaks. ${ }^{7,8)}$ The characteristic findings of cranial magnetic resonance (MR) imaging are diffuse pachymeningeal gadolinium enhancement, subdural fluid collections, sagging of the brain, pituitary hyperemia, and engorgement of venous structure. Most patients with SIH improve spontaneously with bed rest and hydration. If conservative treatment is ineffective, epidural blood patching (EBP) is the treatment of choice. ${ }^{1,7,8)}$ Complications of EBP have been reported in few cases. ${ }^{4,8)}$

We describe a rare case of SIH complicated by delayed subdural hematoma (SDH) with mass effect for which surgical evacuation was required 2 months after EBP.

\section{Case Report}

A 51-year-old woman presented to our hospital with a onemonth history of orthostatic headache. She also reported having nausea, vertigo, and neck pain. Her symptoms worsened when she stood or sat, and were alleviated when she lay down. She had no history of head or spinal trauma. The patient was referred from a community hospital where SIH was diagnosed. She had undergone EBP twice

Received July 23, 2009;

Accepted November 18, 2009 at the L4-5 level at the community hospital.

On her first admission to our hospital, the findings of physical and neurological examinations were normal. Cranial computed tomography (CT) showed bilateral subdural fluid collections. Intrathecal MR cisternography with gadolinium showed CSF leakage site at T12 level (Fig. 1). Cranial $T_{1}$-weighted $M R$ imaging with gadolinium showed diffuse pachymeningeal enhancement, sagging of the brain, and bilateral subdural fluid collections (Fig. 2). Initially, EBP was performed at the L4-5 level. However, blood could not flow to the cephalic side smoothly because of epidural adhesion. EBP was performed at the T12-L1
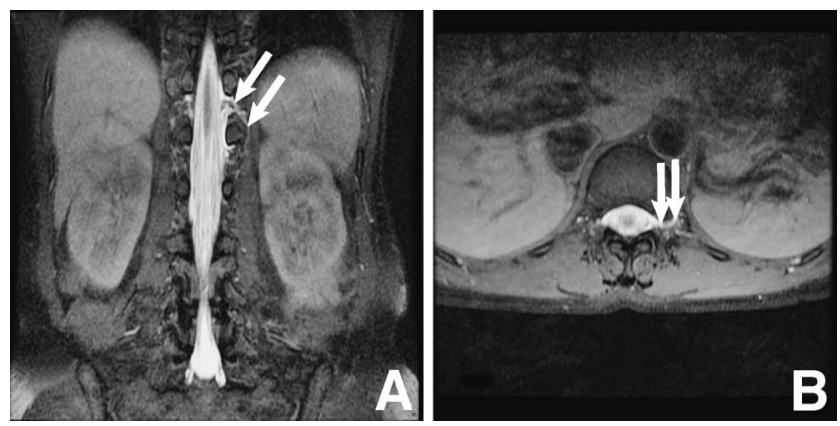

Fig. 1 Coronal (A) and axial (B) magnetic resonance cisternograms showing cerebrospinal fluid leaks (arrows) at the T12 level. 


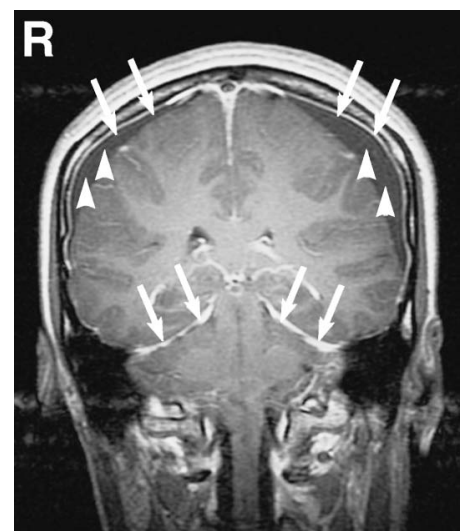

Fig. 2 Coronal $T_{1}$-weighted magnetic resonance image with gadolinium showing a thin layer of subdural effusion on the right (arrowheads), large subdural effusion on the left (arrowheads), and diffuse pachymeningeal enhancement (arrows) over the convexity and tentorium before epidural blood patching.

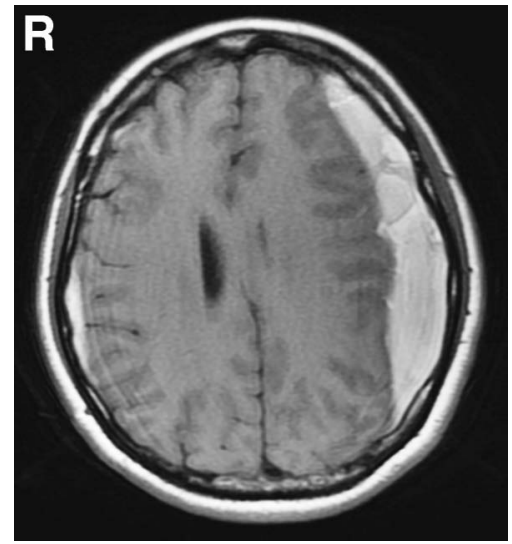

Fig. 3 Axial $\mathrm{T}_{1}$-weighted magnetic resonance image showing a thin layer of chronic subdural hematoma (SDH) on the right and increasing size of the SDH with mass effect on the left 2 months after epidural blood patching.

level with $20 \mathrm{~cm}^{3}$ blood under fluoroscopic guidance. The spread of blood within the epidural space from the lower to upper thoracic levels was confirmed by forward replacement of epidural contrast medium. After EBP, the orthostatic headache subsided. Cranial MR imaging performed 1 week later showed persistent bilateral subdural fluid collections. The patient was discharged home without headache.

Two months later, she returned with increasingly severe headache that was not related to position. Tracing the history carefully, the patient denied any head injury after the first discharge from our hospital. Cranial MR imaging showed enlarged SDH on the left with significant mass effect (Fig. 3). Urgent drainage of the SDH was undertaken through left side frontal burr holes. The patient made an uneventful recovery and remained well. Eighteen months

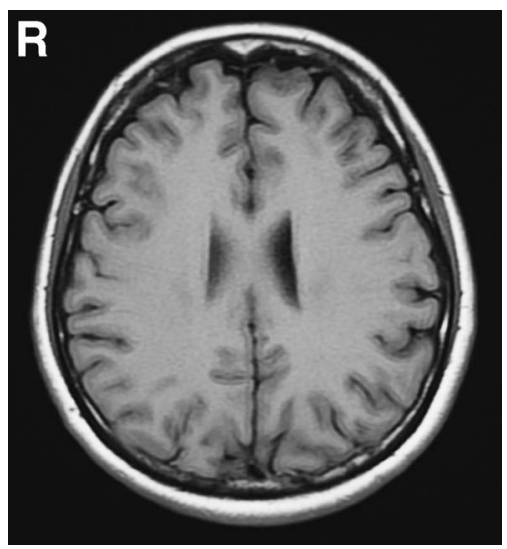

Fig. 4 Axial $T_{1}$-weighted magnetic resonance image showing no subdural fluid or hematoma on both sides 18 months after surgical evacuation of the left chronic subdural hematoma.

after operation, follow-up MR imaging revealed complete resolution of the subdural fluid collection on the right and the SDH on the left (Fig. 4). The patient reported no recurrent headaches during the follow-up period.

\section{Discussion}

SIH is characterized by low intracranial pressure resulting from CSF leaks without a history of dural puncture, surgery, or penetrating injury. Most spontaneous CSF leaks occur at the level of the spine, particularly the thoracic spine. The precise cause of such spontaneous CSF leaks remains unclear, but underlying structural weakness of the spinal meninges is suspected. Dural weakness predisposes the patient to the formation of dural defects that allow CSF to leak into the epidural space. ${ }^{7,8)}$

Most patients with SIH have postural headache, which spontaneously resolves within a few days. However, some patients may report severe symptoms, including progressive postural headache, nausea, vomiting, and dizziness. Rare manifestations include cranial nerve palsies, dural sinus thrombosis, and coma. ${ }^{3,6,9)} \mathrm{SIH}$ is an increasingly common syndrome. However, the diagnosis of SIH is difficult to make based on only cranial CT. If SIH is suspected, several diagnostic tools can be used to establish the diagnosis, including lumbar puncture, radioisotope cisternography, MR imaging, and CT myelography. ${ }^{7-9)} \mathrm{MR}$ imaging of the brain and spine are the first choice. Typical cranial MR imaging findings that suggest SIH include diffuse pachymeningeal enhancement, subdural fluid collections, sagging of the brain, pituitary hyperemia, and engorgement of venous structures. ${ }^{1,8)}$ MR cisternography can detect CSF leaks, ${ }^{1}{ }^{1}$ as in the present case. MR cisternography may show the leakage site of CSF very well, and is superior to radioisotope cisternography and CT myelography because of the higher contrast resolution and sensitivity for small leaks and multiple dural tears. ${ }^{11}$ Additionally, MR cisternography carries no risk from radiation, and multiplanar imaging is possible.

Several treatments have been used for patients with 
SIH, including bed rest, hydration, caffeine, EBP, fibrin glue injection, intrathecal saline injection, and surgical repair of the leak. ${ }^{2,4,5)}$ In the present case, medical treatment and EBP performed twice at the L4-5 level failed to correct the SIH. Initially, we performed EBP at the L4-5 level, but failed because of epidural adhesion, so we changed the EBP injection site to the T12-L1 junction under fluoroscopic guidance. EBP under fluoroscopic guidance is useful for identifying individual variations in the spread of the blood. Repeat EBP may be needed, as in $56 \%$ of patients in one series. ${ }^{2)}$ EBP can cause epidural adhesion, spinal nerve damage, chemical meningitis, rebound intracranial hypertension, and inadvertent dural puncture. Most patients with SIH who undergo EBP have a good outcome..$^{2,45)}$ Improvement of MR imaging abnormalities can be seen within hours to weeks after successful treatment of the CSF leak.

$\mathrm{SIH}$ with subdural fluid collection has been reported in $10-69 \%$ of patients. ${ }^{3,6,9)} \mathrm{SDH}$ was reported in $40-47 \%$ of patients with subdural fluid collection. ${ }^{3,6,9)}$ The pathogenesis of SDH remains unclear, but may involve tearing of bridging veins or bleeding from enlarged veins in the subdural space because of low intracranial pressure and brain descent. ${ }^{3,6)}$ In addition, meningeal biopsy results have suggested that the fragile and dilated new dural vessels in $\mathrm{SIH}$ patients might account for SDH, even after normal intracranial pressure has returned. ${ }^{3)}$ Subdural fluid collections associated with $\mathrm{SIH}$ including large $\mathrm{SDH}$ associated with significant mass effect can be safely managed by directing treatment at the underlying CSF leak without the need for craniotomy or burr hole drainage. ${ }^{6,9)}$ Large SDH may require up to 3 months to resolve after treatment of the spinal CSF leak. ${ }^{8)}$ Only a few reports have recommended evacuation for patients with progressive development of SDH. ${ }^{3,6,9)}$ However, in the present case, subdural fluid collection did not resolve after treatment of the spinal CSF leak, and even caused delayed SDH with mass effect 2 months after successful EBP, finally requiring surgical evacuation.

The present case of SIH was complicated by delayed
SDH after EBP. The clinical course and imaging findings of this patient provide some insight into the pathophysiology of SIH and illustrate the possible sequential complications of EBP.

\section{References}

1) Albayram S, Kilic F, Ozer H, Baghaki S, Kocer N, Islak C: Gadolinium-enhanced MR cisternography to evaluate dural leaks in intracranial hypotension syndrome. AJNR Am J Neuroradiol 29: 116-121, 2008

2) Berroir S, Loisel B, Ducros A, Boukobza M, Tzourio C, Valade D, Bousser MG: Early epidural blood patch in spontaneous intracranial hypotension. Neurology 63: 1950-1951, 2004

3) de Noronha RJ, Sharrack B, Hadjivassiliou M, Romanowski CA: Subdural haematoma: a potentially serious consequence of spontaneous intracranial hypotension. J Neurol Neurosurg Psychiatry 74: 752-755, 2003

4) Ishikawa S, Yokoyama $M$, Mizobuchi $S$, Hashimoto $H$, Moriyama E, Morita K: Epidural blood patch therapy for chronic whiplash-associated disorder. Anesth Analg 105: 809-814, 2007

5) Kong DS, Park K, Nam DH, Lee JI, Kim JS, Eoh W, Kim JH: Clinical features and long-term results of spontaneous intracranial hypotension. Neurosurgery 57: 91-96, 2005

6) Lai TH, Fuh JL, Lirng JF, Tsai PH, Wang SJ: Subdural haematoma in patients with spontaneous intracranial hypotension. Cephalalgia 27: 133-138, 2007

7) Mokri B: Headaches caused by decreased intracranial pressure: diagnosis and management. Curr Opin Neurol 16: 319326, 2003

8) Schievink WI, Maya MM: Spontaneous spinal cerebrospinal fluid leaks. Headache 48: 1511-1519, 2008

9) Schievink WI, Maya MM, Moser FG, Tourje J: Spectrum of subdural fluid collections in spontaneous intracranial hypotension. J Neurosurg 103: 608-613, 2005

Address reprint requests to: Han-Jung Chen, M.D., Ph.D., Department of Neurosurgery, E-Da Hospital, I-Shou University, 1 E-Da Road, Yan-Chau Shiang, Kaohsiung County 824, R.O.C.

e-mail: ed100183@edah.org.tw 